\title{
Trend modelling of wave parameters and application in onboard prediction of ship
} responses

Montazeri, Najmeh; Nielsen, Ulrik Dam; Jensen, J. Juncher

Published in:

Proceedings of OCEANS '15

Publication date:

2015

Document Version

Peer reviewed version

Link back to DTU Orbit

Citation (APA):

Montazeri, N., Nielsen, U. D., \& Jensen, J. J. (2015). Trend modelling of wave parameters and application in onboard prediction of ship responses. In Proceedings of OCEANS '15 [7401961] IEEE.

\section{General rights}

Copyright and moral rights for the publications made accessible in the public portal are retained by the authors and/or other copyright owners and it is a condition of accessing publications that users recognise and abide by the legal requirements associated with these rights.

- Users may download and print one copy of any publication from the public portal for the purpose of private study or research.

- You may not further distribute the material or use it for any profit-making activity or commercial gain

- You may freely distribute the URL identifying the publication in the public portal

If you believe that this document breaches copyright please contact us providing details, and we will remove access to the work immediately and investigate your claim. 


\section{Trend modelling of wave parameters and application in onboard prediction of ship responses}

\author{
Najmeh Montazeri \\ Department of \\ Mechanical Engineering,

\begin{abstract}
This paper presents a trend analysis for prediction of sea state parameters onboard ships during voyages. Given those parameters, a JONSWAP model and also the transfer functions, prediction of wave induced ship responses are thus made. The procedure is tested with full-scale data of an in-service container ship. Comparison between predictions and the actual measurements, implies a good agreement in general. This method can be an efficient way to improve decision support onboard ships.
\end{abstract}

Ulrik D. Nielsen

a) Department of

Mechanical Engineering,
NTNU,Norway

Email: udn@mek.dtu.dk

Technical University of Denmark Technical University of Denmark Technical University of Denmark

J. Juncher Jensen

a) Department of

Mechanical Engineering,

Email: najmon@mek.dtu.dk b) Centre for Autonomous Marine b) Centre for Autonomous Marine Operations and Systems(AMOS), Operations and Systems(AMOS),

NTNU,Norway

Email: jjj@mek.dtu.dk

\section{INTRODUCTION}

The concept of decision support system for safe operation of ships has been of interest in research for many years. In particular, providing information about the ship's wave induced loads and seakeeping within near future is very useful in such a way that excessive responses can be avoided by making decision on the course and the speed of the ship. In order to get such prediction information, an estimate of the actual sea state should be available. Different tools exist for on-site sea state estimation. This estimation can also be carried out based on ship responses themselves; in a way similar to that of traditional wave rider buoys. Different approaches in this field can be found in the literature e.g.[14]. Several researches have also been carried out for prediction of wave induced responses, usually represented as standard deviation of the responses e.g.[5-7]. This information can be monitored in a graphical user interface to provide onborad decision support [8].

The wave estimation method used in this paper is based on the approach in $[9,10]$; a study on optimisation of parametric wave spectrum through the spectral moments of ship responses. Analysis of full-scale measurements of a large in-service container ship is carried out herein as a case study for the presented estimation and prediction approaches.

\section{Moment-BAsed WAVE EStimation}

A JONSWAP wave spectrum model is considered for real-time estimation of incoming waves using measurements and transfer functions of different responses of the ship. As mentioned before, the optimisation of the sea state parameters is based on spectral moments of the response spectra as follows:

$$
\begin{aligned}
& \int_{\omega_{e_{l}}}^{\omega_{e_{h}}} \Phi e_{i j}\left(\omega_{e}\right) d \omega_{e}= \\
& \int_{\omega_{l}}^{\omega_{h}} \int_{-\pi}^{\pi} H_{i}(\omega, \theta) H_{j}^{*}(\omega, \theta) S(\omega, \theta) d \theta d \omega \\
& \quad i, j=1,2, \ldots, N
\end{aligned}
$$

where $\omega$ is frequency, $\theta$ is direction, $S$ is the directional wave spectrum and $\Phi e_{i j}$ is cross spectral 
TABLE I: Ship characteristics

\begin{tabular}{ll}
\hline Properties & Values \\
\hline Overall Length [m] & 349.0 \\
Beam [m] & 42.8 \\
Max. Draft [m] & 15.0 \\
Max. Capacity [TEU] & 9415 \\
DWT [ton] & 113,000 \\
\hline
\end{tabular}

density of $i$ th and $j$ th responses in the encounter frequency domain. $H$ denotes the complex-valued transfer function, ${ }^{*}$ is the conjugate notation and $N$ is the number of responses. In this method the wave spectrum should be split into two; a -low frequency and a high frequency- part using Eq.(1) for each part. $\omega_{l}$ and $\omega_{h}$ determine the limits of frequencies for each partition. The left-hand side of Eq.(1) is measured in encounter frequency domain whereas the right-hand side is the theoretical spectra calculated in wave frequency domain [9].

\section{CASE STUDY}

The main characteristics of the vessel are given in Table I. There are two other systems already installed onboard this ship to estimate the sea state: 1) an X-band radar system (WaMoS) which scans the sea surface and provides sea state parameters (the average period, the significant wave height and the relative wave direction), and 2) wave guide system (Radac) which provides the absolute (estimated) wave height based on measurements of relative wave height combined with the absolute displacement derived from double-integrated vertical acceleration [11]. These sources of wave data are used here for comparison with the calculated spectra based on ship responses. Hindcast data is also available from Deutscher Wetterdienst for typical locations and times.

Full scale data for 4 days of operation in 2011 is considered. The operational conditions and the geographical locations are specified in Table II. The time series of vertical motion, pitch, sway and vertical bending moment are used to estimate the wave parameters in JONSWAP spectrum $(S$ in Eq.(1)). The main three parameters are the significant wave height, the peak period and the relative wave direction.
TABLE II: Operational conditions

\begin{tabular}{llll}
\hline Cases & Draft $[\mathrm{m}]$ & Speed[kn] & Location \\
\hline I & 14.2 & $21.0-23.5$ & Gulf of Aden \\
II & 14.0 & $17.0-18.0$ & Gulf of Aden \\
III & 14.0 & $11.5-13.5$ & South of India \\
IV & 15.0 & $9.5-14.0$ & Off Hong Kong \\
\hline
\end{tabular}

In practice, wave systems are assumed to be stationary in a duration of the order of 20-60 minutes. In the current study of response based wave estimation, as the geographical location of ship is subject to change continuously, the optimisation is implemented based on 20 minutes long data as a stationary period for wind waves; however this assumption might be idealistic in severe sea states where the conditions can change quickly. On the other hand, swell events seem to be stationary for a longer period, say 1-2 hours.

The estimations are carried out in segments of 20 minutes. The average wind speed and the average wind direction within 20 minutes are used. Following the same procedure as in [9], first, a single wave spectrum is fitted to the measured data using the accelerations and the displacements of the mentioned responses. Then the estimated peak frequency is compared to the peak of PiersonMoskowitz, PM, spectrum which is calculated based on the average value of the measured wind speed [12].

$$
\omega_{P M}=0.82 \frac{g}{U_{w}}
$$

where $U_{w}$ is usually $10 \mathrm{~m}$ elevation wind speed in $\mathrm{m} / \mathrm{s}$. If $\omega_{p}<\omega_{P M}$, the waves are considered as swell dominated and if $\omega_{p}>\omega_{P M}$ they are wind dominated. Partitioning is then applied by separating the wind (high frequency) part from the swell (low frequency) part. For the considered cases in Table II, however, no bimodality was found by this approach. As discussed in [9], apart from wind speed, the measured wind direction with an interval, \pm 90 deg., is considered as a constraint for optimisation of the relative direction of wind seas. The estimated parameters are presented in section $\mathrm{V}$. 


\section{TREND MODELLING OF WAVE PARAMETERS}

In order to provide a more efficient usage of estimated wave parameters, an automatic dynamic model is introduced in this section to evaluate the tendency of these parameters during the voyage. This type of results is of interest to upgrade onboard response predictions. Moreover, available estimates of the trends could provide initial guesses and proper constraints as inputs to estimation procedure of the sea state itself. A general form of regression model is expressed as:

$$
Y_{t}=\boldsymbol{x}_{\boldsymbol{t}} \boldsymbol{\theta}+\epsilon_{t}
$$

where $Y_{t}$ is a dependent variable, $x_{t}$ is a known vector of independent variables and $\boldsymbol{\theta}=\left(\theta_{1}, \ldots, \theta_{p}\right)^{T}$ is a vector of model parameters which are called regression parameters hereafter to be distinguished from the wave parameters. The residual, $\epsilon_{t}$, is a random variable with $E\left[\epsilon_{t}\right]=0$ and $\operatorname{Var}\left[\epsilon_{t}\right]=\sigma^{2}$. Although both time and location change during a voyage, only time variations of waves are considered herein. Therefore, the index $t$ denotes the variables at a time scale $t$ and a certain function of time, $\boldsymbol{x}_{\boldsymbol{t}}=f^{T}(t)$, is considered for this independent variable. Thus, when $N$ observations are available, the implemented model reads [13]:

$$
Y_{N+t}=f^{T}(t) \boldsymbol{\theta}+\epsilon_{N+t}
$$

Amongst the mathematical functions, a $p$ th-order polynomial is widely used for $f(t)[13,14]$. A quadratic model with $p=3$ parameters is considered here:

$$
Y_{N+t}=\theta_{0}+\theta_{1} t+\theta_{2} \frac{t^{2}}{2}+\epsilon_{N+t}
$$

using $f(t)=\left(1, t, \frac{t^{2}}{2}\right)^{T}$.

The vector of regression parameters is estimated by least square method given the observations $Y_{1}, \ldots, Y_{N}$. A local trend model is used where the observations in the far past are given less weight than the recent observations, in the least square criterion. This is carried out by considering a forgetting factor, $\lambda(|\lambda|<1)$, which determines the discount of past observations. The sum of squared residuals are expressed as:

$$
S S R(\boldsymbol{\theta} ; N)=\sum_{t=0}^{N-1} \lambda^{t}\left[Y_{N-t}-f^{T}(-t) \boldsymbol{\theta}\right]^{2}
$$

The vector of regression parameters at time $N, \widehat{\boldsymbol{\theta}}_{N}$, is $\boldsymbol{\theta}$ that minimizes $S S R(\boldsymbol{\theta} ; N)$ in Eq.(6). By using weighted least square method, it is obtained as [13]:

$$
\widehat{\boldsymbol{\theta}}_{N}=F_{N}^{-1} h_{N}
$$

where

$$
F_{N}=\sum_{t=0}^{N-1} \lambda^{t} f(-t) f^{T}(-t)
$$

and

$$
h_{N}=\sum_{t=0}^{N-1} \lambda^{t} f(-t) Y_{N-t}
$$

Estimation of the parameters is updated at each time step when the next observation $Y_{N+1}$ is available. The forgetting factor is applied here as $\lambda=0.85$. The prediction of $Y$ given the observations at time step $N, \widehat{Y}_{N+l \mid N}$, is calculated by:

$$
\widehat{Y}_{N+l \mid N}=f^{T}(l) \widehat{\boldsymbol{\theta}}_{N}
$$

An interval is usually defined around the predicted value as a confidence level or a coverage probability which is called prediction interval. A $100(1-\alpha) \%$ prediction interval is calculated as:

$$
\widehat{Y}_{N+l \mid N} \pm t_{\alpha / 2}(N-p) \sqrt{\operatorname{Var}\left[e_{N}(l)\right]}
$$

where $t_{\alpha / 2}(N-p)$ is the $\alpha / 2$ quantile in the $\mathrm{t}$ (Student's) distribution. The variance of the prediction error, $e_{N}(l)=Y_{N+l}-\widehat{Y}_{N+l \mid N}$, is:

$$
\operatorname{Var}\left[e_{N}(l)\right]=\sigma^{2}\left[1+f^{T}(l) F_{N}^{-1} f(l)\right]
$$

where $\sigma^{2}$ should be estimated by:

$$
\widehat{\sigma}^{2}=\frac{\sum_{t=0}^{N-1} \epsilon_{t}}{N-p}
$$

For more details on the mathematical model see [13].

The full scale data from Sec. II are used to model the trends of estimated wave parameters. The predictions are started when 3 hours data are available. 


\section{RESUlts AND Discussion}

Figure 1 shows the estimated wave peak periods and the prediction trends during 24 hours of the considered cases being compared with WaMoS data. For the whole day of case No.I, the estimated peak frequency is higher than the peak of PM spectrum (Eq.(2)) implying that the waves are wind dominated. Since no swell event is recognized in the low frequency fit, it is assumed thereby that the wave spectrum is unimodal. The magnitudes of the peak periods conform very well with WaMoS except the last few hours where the predicted peak periods fall around $6 \mathrm{~s}$.

In case No.II, the estimations imply swell dominated spectra since the estimated peak frequencies are lower than the corresponding peak of the PM spectrum. In the last quarter of the day (time 1923 ), the estimated peak frequencies are very close to the peak of the PM spectrum. Thus, it is probably reasonable to identify the waves in this period as fully developed sea. By implementing the partitioning approach, no wind sea is pronounced during this day.

In case No.III the waves are also swell dominated. The measured wind speed is very low so that the separation frequency is very high and no wind sea is identified. The predicted values in the cases II and III stand consistently above the WaMoS estimations. It is likely that the high frequency part of the spectrum is filtered out by the ship.

The waves in case No.IV are assigned as wind dominated since the estimated peak frequencies always fall considerably higher than the peak frequency of PM spectrum. The secondary wave system in the low frequency part is neglected because of very small amplitudes. Although the predictions are slightly lower than the WaMoS estimates, the agreement is acceptable.

Figure 2 shows the estimated significant wave heights and the prediction trends being compared with the estimated values from WaMoS and Radac. As seen in this figure, all sources are to some extent in agreement, designating case I to moderate sea, case II to mild and moderate sea, case III to mild sea and case IV to dominantly severe sea states.

The trends of predictions follow very well with
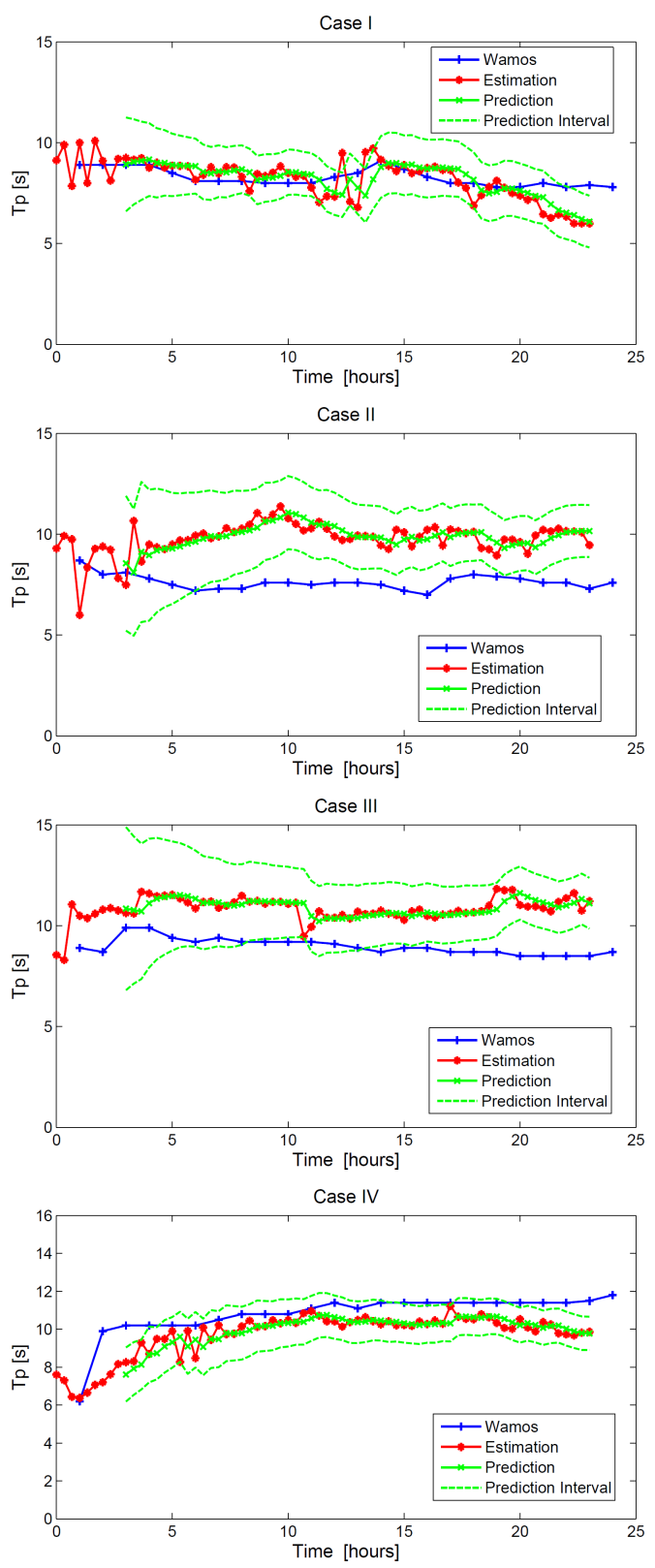

Fig. 1: Wave peak period

the tendency of WaMoS and Radac data during the operational days. However, from quantitative point of view, underestimation up to 2 meters are seen in some periods compared to WaMoS. In the afternoon hours in case No.IV, a bias deviation can 

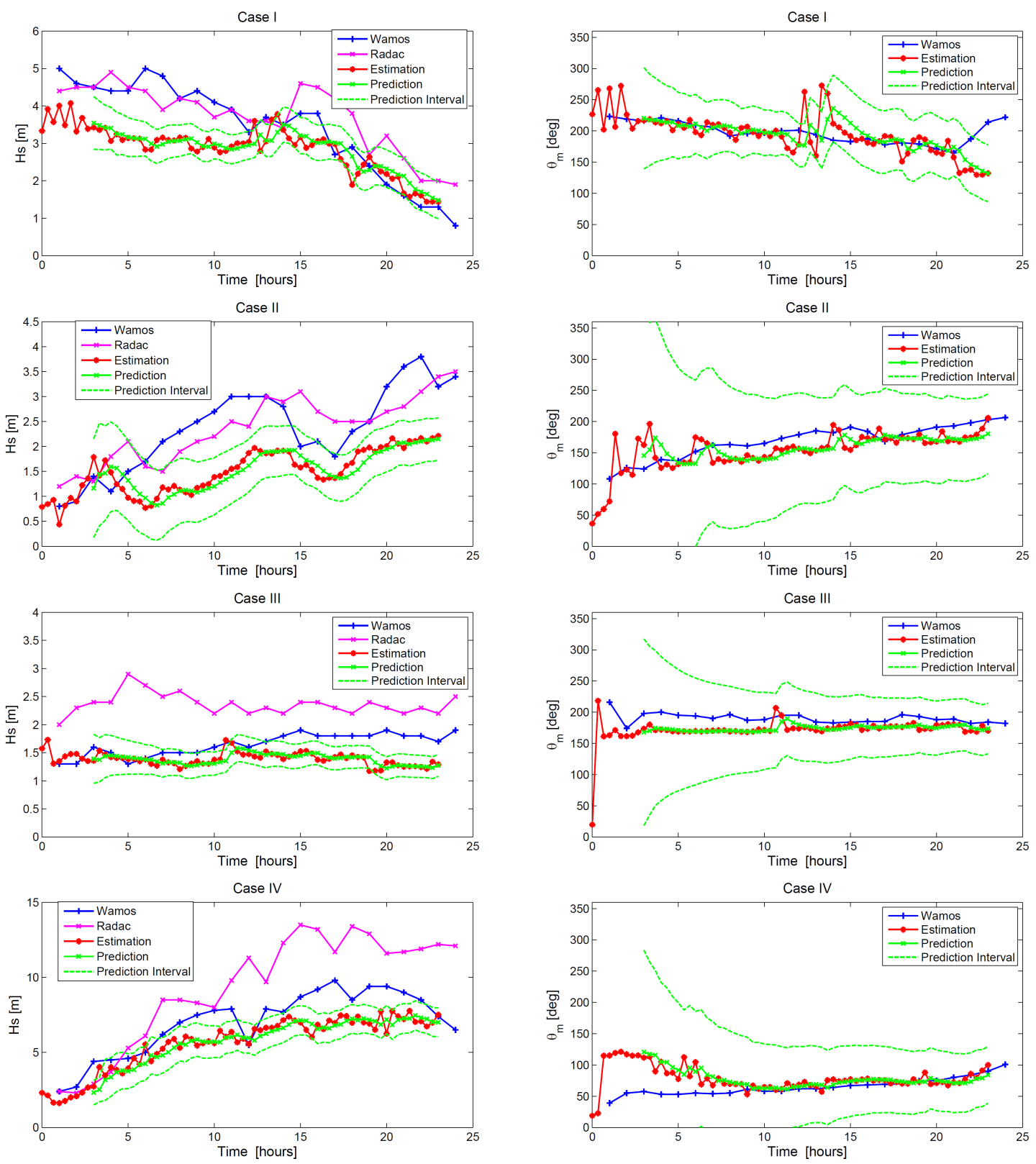

Fig. 2: Significant wave height

also be observed between predictions and Radac which exceeds 6.5 meters. It is not easy to judge which data is more accurate in this period. But since the WaMoS data is also very different from Radac and because of sprays of water in the bow or due

to wave-hall disturbances, it is likely that the Radac measurements are erroneous specially in this severe wave condition.

In order to apply the trend analysis on estimated wave directions, the ship's heading should be sub- 
tracted from the relative directions. So the predicted mean wave directions toward north are illustrated in figure 3. As seen in this figure, the estimated wave directions agree well with WaMoS except an error up to $60 \mathrm{deg}$. that is observed in the first few hours of case IV. As mentioned before, a signal period of 1 hour is considered in swell event estimations i.e. cases II and III. This consideration gives more consistent estimates for wave directions.

Based on Figures 1-3, it can be concluded that beside the advantage of making predictions 20 minutes ahead of estimations, the trend analysis, provides smooth evolution curves for wave parameters. In this way, probable erroneous outliers in the estimations can be compensated since the local sea state is not likely to change quickly in short time. This can be implemented through proper initialization in the optimisation of the wave parameters. Moreover, the prediction intervals can be used as constraints to avoid erratics observed e.g. in the first few hours of cases I and II.

\section{Vi. Prediction of Responses}

Typically, real-time estimation of responses, say 20-30 minutes ahead of measurements, assumes the sea state to be stationary, with a possible change in only operational parameters (vessel speed and heading). As shown and discussed in [5], these assumptions are, however, not necessarily valid. Therefore, to improve the precision and reliability of response predictions, the present study suggests to adopt the predicted magnitudes of the wave parameters from the trend analysis in Sec.IV. These values together with information about the new operational condition can be used to predict the responses.

This procedure is applied here to predict the motions and the vertical bending moment. A combination of JONSWAP wave model with the predicted parameters from Sec.V and the transfer functions are used again to obtain the responses:

$$
R_{i}=\int_{0}^{\infty} \int_{-\pi}^{\pi}\left|H_{i}(\omega, \theta)\right|^{2} S(\omega, \theta) d \theta d \omega
$$

where $R_{i}$ is the 0th spectral moment (or the variance) of the $i$ th response which represents the
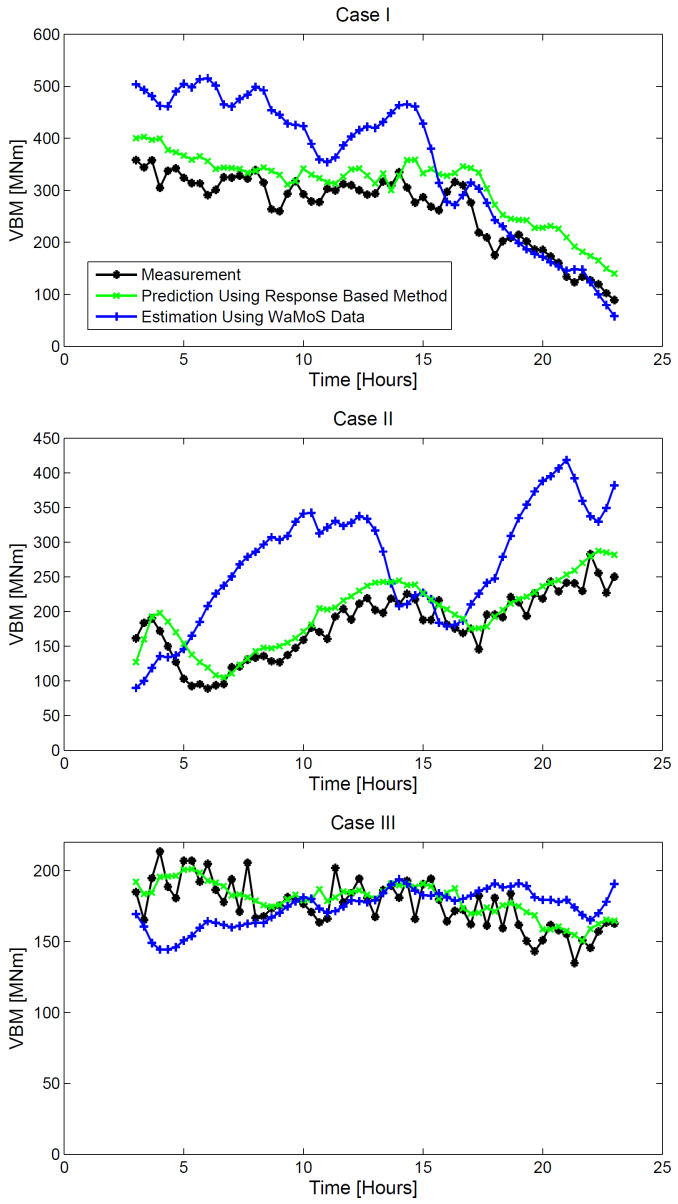

Case IV

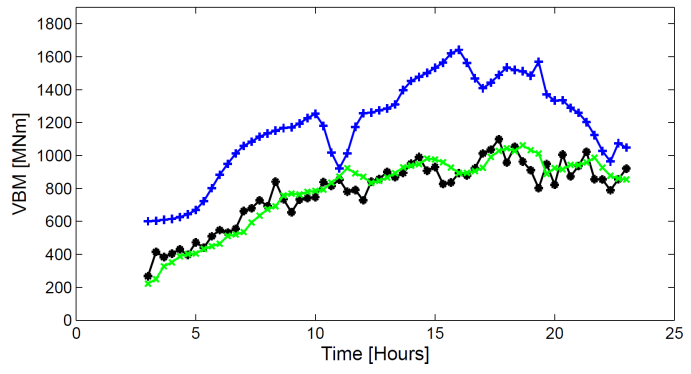

Fig. 4: Standard deviation of Vertical Bending Moment (The legends are identical in all plots)

energy amount of that response. Similar to Sec.V the predictions are made 20 minutes ahead of measurements.

The square roots of the predicted variances i.e. 

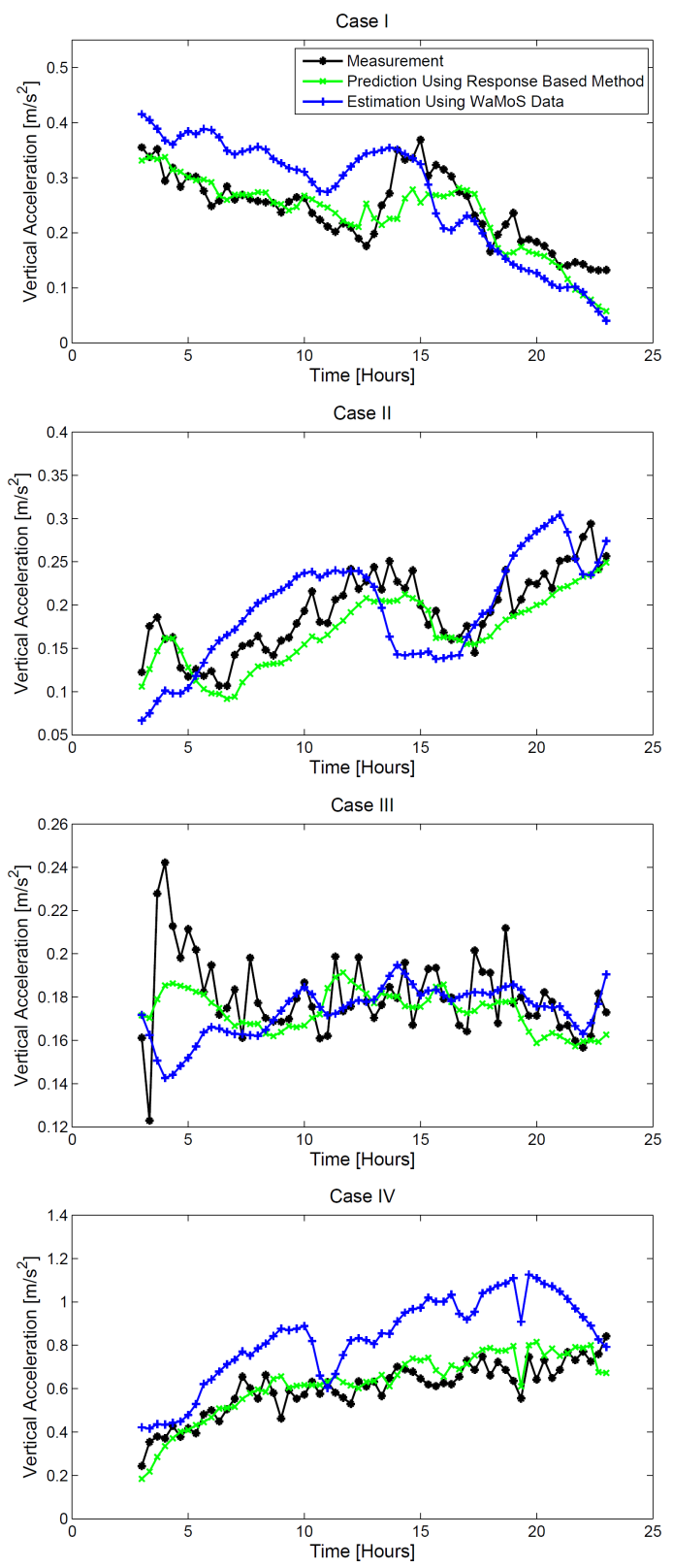

Fig. 5: Standard deviation of Vertical Acceleration

(The legends are identical in all plots)

the standard deviations are compared with corresponding measured signals. Figure 4 shows this comparison for the vertical bending moment at midship section which is extracted from stress mea- surements. The predictions are in general in a good agreement with the real measurements. However, an error up to $50 \mathrm{MNm}$ can be observed in case I. The blue curves show the estimated responses based on WaMoS data using also a JONSWAP model. Although the trends of WaMoS based estimations are in qualitative agreement with the predictions, some quantitative differences exist which could be due to either the response measurement errors, WaMos measurement errors or filtering effects of the ship.

Figure 5 shows the vertical acceleration at midship section on the port side which is a combination of heave and roll accelerations. The magnitudes and the trends of predictions are in good agreements with the measurements. It can be seen in this figure that the predictions mostly conform with the WaMoS based estimations as well, except in a few cases where the waves contain relatively large amounts of energy.

Although not shown in this paper, it was observed that the predicted roll motions are very different from the measurements in all cases. It is believed that those errors in this motion are dominantly due to the measurements since the amplitudes are sometimes unexpectedly large compared to the magnitudes of roll transfer functions in typical operational conditions.

\section{CONCLUSION}

A local regression trend model is proposed for prediction of sea state parameters. A wave estimation analysis is carried out using full scale data of a large container ship. This approach is based on the moments of response spectra and strengthens the validity of the study in [9]. However the trend model is applicable to any sea state data, no matter which estimation method is used. The predictions are made in a time horizon of 20 minutes. These trend models provide a smooth and consistent evolution of wave parameters which is more realistic as the sea state varies quite slowly in time. The model can also associate sort of confidence bands to predictions.

The predictions are then used as input to estimate the future responses of the ship using a JONSWAP 
model, the updated operational condition, and the corresponding transfer functions. The results show a good agreement between the predictions and the actual measurements. This concept, in combination with the procedure for response prediction given in [5], could be useful for development of decision support system particularly for operational safety by avoiding undue loads, motions or accelerations.

\section{ACKNOWLEDGMENT}

The authors sincerely acknowledge Class NK for supporting the research project in cooperation with the Technical University of Denmark.

\section{REFERENCES}

[1] E.A. Tannuri, J.V. Sparano, A.N. Simos, and J.J. Da Cruz. Estimating directional wave spectrum based on stationary ship motion measurements. Applied Ocean Research, 25(5):243-261, October 2003.

[2] R. Pascoal and C. Guedes Soares. Kalman filtering of vessel motions for ocean wave directional spectrum estimation. Ocean Engineering, 36(6-7):477-488, May 2009.

[3] U.D. Nielsen and D.C. Stredulinsky. Sea state estimation from an advancing ship A comparative study using sea trial data. Applied Ocean Research, 34:33-44, January 2012.

[4] U.D. Nielsen. The wave buoy analogy estimating high-frequency wave excitations. Applied Ocean Research, 30(2):100-106, April 2008.

[5] U.D. Nielsen and T. Iseki. Prediction of FirstOrder Vessel Responses with Applications to Decision Support Systems. In 5th World Maritime Technology Conference, 2015.

[6] M. Huss and A. Olander. Theoretical Seakeeping Predictions On Board Ships - A System for Operational Guidance and Real Time Surveillance. Technical report, Naval Architecture, Department of Vehicle Engineering, Royal Institute of Technology, 1994.

[7] H. G. Payer and H. Rathje. Shipboard Routing Assistance Decision Making Support for Operation of Container Ships in Heavy Seas. Trans. of SNAME, 112:1-12, 2004.
[8] J.K. Nielsen, N.H. Hald, J. Michelsen, U.D. Nielsen, J. Baatrup, J.J. Jensen, and E.S. Petersen. SeaSense Real-time Onboard Decision Support. In World Maritime Technology Conference, 2006.

[9] N. Montazeri, U.D. Nielsen, and J.J Jensen. Estimation of wind sea and swell using shipboard measurements-A refined parametric modelling approach. Applied Ocean Research (under review), 2015.

[10] N. Montazeri and U.D. Nielsen. Parametric estimation in the wave buoy analogy - an elaborated approach based on energy considerations. In Proceedings of the ASME 33rd International Conference on Ocean, Offshore and Arctic Engineering (OMAE), 2014.

[11] U.D. Nielsen, I.M.V. Andersen, and J. Koning. Comparisons of Means for Estimating Sea States from an Advancing Large Container Ship. In Proceeding of the PRADS 2013. PRADS, 2013.

[12] J. Portilla, F. J. Ocampo-Torres, and J. Monbaliu. Spectral Partitioning and Identification of Wind Sea and Swell. Journal of Atmospheric and Oceanic Technology, 26(1):107122, January 2009.

[13] H. Madsen. Time Series Analysis. CRC Press, 2007.

[14] G Kitagawa. Introduction to time series modeling. 2010. 